\title{
Coherent pulsed excitation of degenerate multistate systems: Exact analytic solutions
}

\author{
E.S. Kyoseva ${ }^{1,2}$ and N.V. Vitanov ${ }^{1,3}$ \\ ${ }^{1}$ Department of Physics, Sofia University, James Bourchier 5 blvd., 1164 Sofia, Bulgaria \\ ${ }^{2}$ Fachbereich Physik der Universität, 67653 Kaiserslautern, Germany \\ ${ }^{3}$ Institute of Solid State Physics, Bulgarian Academy of Sciences, Tsarigradsko chaussée 72, 1784 Sofia, Bulgaria
}

(Dated: November 5, 2018)

\begin{abstract}
We show that the solution of a multistate system composed of $N$ degenerate lower (ground) states and one upper (excited) state can be reduced by using the Morris-Shore transformation to the solution of a two-state system involving only the excited state and a (bright) superposition of ground states. In addition, there are $N-1$ dark states composed of ground states. We use this decomposition to derive analytical solutions for degenerate extensions of the most popular exactly soluble models: the resonance solution, the Rabi, Landau-Zener, Rosen-Zener, Allen-Eberly and Demkov-Kunike models. We suggest various applications of the multistate solutions, for example, as tools for creating multistate coherent superpositions by generalized resonant $\pi$-pulses. We show that such generalized $\pi$-pulses can occur even when the upper state is far off resonance, at specific detunings, which makes it possible to operate in the degenerate ground-state manifold without populating the (possibly lossy) upper state, even transiently.

PACS numbers: 32.80.Bx, 32.80.Qk, 33.80.Be, 32.80.-t, 33.80.-b
\end{abstract}

\section{INTRODUCTION}

The problem of a two-state quantum system driven by a time-dependent pulsed external field plays a central role in quantum physics [1]. First of all, this problem is interesting by itself both physically and mathematically: physically, because the two-state system is the simplest nontrivial system with discrete energy states in quantum mechanics; mathematically, because the Schrödinger equation for two states poses interesting mathematical challenges some of which are exactly soluble. Furthermore, already in the two-state case, important nonclassical phenomena occur, for instance, the famous Rabi oscillations, which often serve as a test for quantum behavior, and also provide a powerful tool for coherent control of quantum dynamics, e.g. by $\pi$ pulses. Finally, in almost all cases (except for a few exactly soluble), the behavior of a multistate quantum system can only be understood by reduction to one or more effective two-state systems, e.g., by adiabatic elimination of weakly coupled states or by using some intrinsic symmetries.

Besides the well-known solution for exact resonance, there exist several exactly soluble two-state models, the most widely used being the Rabi [2], Landau-Zener 3], Rosen-Zener [4], Allen-Eberly [5], Bambini-Berman [6], Demkov-Kunike [7], Carroll-Hioe [8], Demkov [9] and Nikitin [10] models. All these models provide the transition probability between two nondegenerate states.

In the present paper, we present the extensions of these exactly soluble models to the case when one of the states is replaced by $N$ degenerate states, as displayed in Fig. 1. By using the Morris-Shore (MS) transformation [11] we show that the $(N+1)$-state problem can be reduced to an effective two-state problem involving a bright state and the upper, nondegenerate state. If known, the propagator for this subsystem can be used to find the solution for the full $(N+1)$-state system. Such analytic solutions can be very useful in designing general unitary transformations within the $N$-state degenerate manifold, which can be viewed as a qunit for quantum information processing [12]. We point out that the same system for $N=3$ has been considered by Unanyan et al [13] and by Kis and Stenholm [14] for general $N$, who have derived the adiabatic solution for pulses generally delayed in time; these schemes extend the well-known technique of stimulated Raman adiabatic passage (STIRAP) (see [15] for reviews). Here we derive several exact analytic solutions for pulses coincident in time. This work can therefore be considered as an extension to arbitrary $N$ of an earlier paper [17], which treated the case $N=2$.

This paper is organised as follows. In Sec. III we describe the system and define the problem. In Sec. III we introduce the MS basis and derive the $(N+1)$-state propagator in terms of the (presumably known) two-state propagator. In Sec. IV we use this solution to identify various interesting types of population evolutions. In Sec. V we use the analytic solutions for exact resonance and the Rosen-Zener model to propose several applications, for example, creation of maximally coherent superpositions and qunit rotation. In Sec. VI we discuss some aspects of the multistate Landau-Zener and Demkov-Kunike models. Finally, Sec. VII provides a summary of the results.

\section{DEFINITION OF THE PROBLEM}

\section{A. System Hamiltonian}

We consider an $(N+1)$-state system with $N$ degenerate lower (ground) states $\left|\psi_{n}\right\rangle(n=1,2, \ldots, N)$ and one upper (excited) state $\left|\psi_{N+1}\right\rangle$, as depicted in Fig. 11, The $N$ lower states are coupled via the upper state with pulsed interactions, each pair of which are on two- 


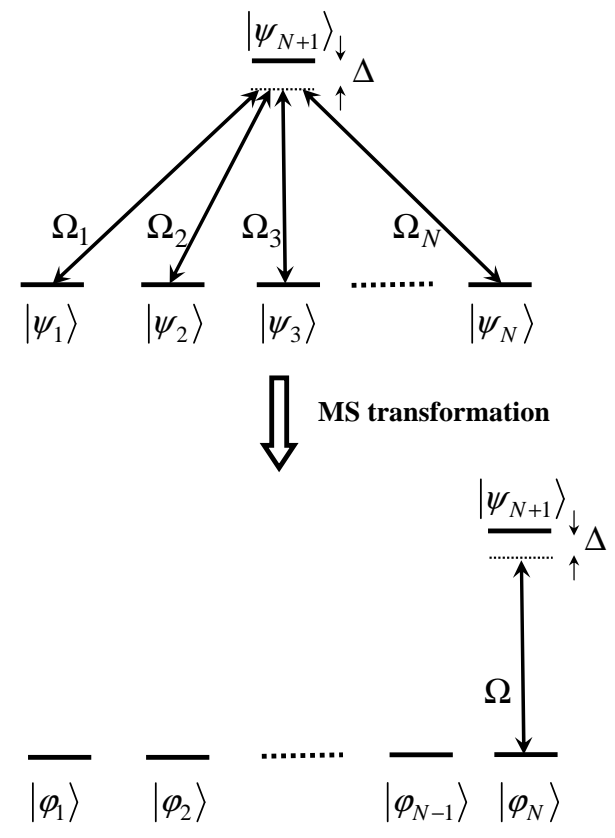

FIG. 1: Top: The system studied in this paper. $N$ degenerate (in RWA sense) states $\left|\psi_{1}\right\rangle,\left|\psi_{2}\right\rangle, \ldots,\left|\psi_{N}\right\rangle$ are coupled simultaneously to an upper state $\left|\psi_{N+1}\right\rangle$, possibly off singlephoton resonance by a detuning $\Delta(t)$, with Rabi frequencies $\Omega_{n}(t)(n=1,2, \ldots, N)$. Bottom: The same system in the Morris-Shore basis. There are $N-1$ uncoupled dark states $\left|\varphi_{1}\right\rangle,\left|\varphi_{2}\right\rangle, \ldots,\left|\varphi_{N-1}\right\rangle$, and a pair of coupled states, a bright state $\left|\varphi_{N}\right\rangle$ and the upper state $\left|\psi_{N+1}\right\rangle$, with the same detuning $\Delta(t)$ as in the original basis and a coupling given by the rms Rabi frequency $\Omega(t)$, Eq. (6).

photon resonance (Fig. 1). The upper state $\left|\psi_{N+1}\right\rangle$ may be off single-photon resonance by some detuning $\Delta(t)$ that, however, must be the same for all fields. In the usual rotating-wave approximation (RWA) the Schrödinger equation of the system reads [1]

$$
i \hbar \frac{d}{d t} \mathbf{C}(t)=\mathbf{H}(t) \mathbf{C}(t)
$$

where the elements of the $(N+1)$-dimensional vector $\mathbf{C}(t)$ are the probability amplitudes of the states and the Hamiltonian is given by

$$
\mathbf{H}(t)=\frac{\hbar}{2}\left[\begin{array}{ccccc}
0 & 0 & \cdots & 0 & \Omega_{1}(t) \\
0 & 0 & \cdots & 0 & \Omega_{2}(t) \\
\vdots & \vdots & \ddots & \vdots & \vdots \\
0 & 0 & \cdots & 0 & \Omega_{N}(t) \\
\Omega_{1}(t) & \Omega_{2}(t) & \cdots & \Omega_{N}(t) & 2 \Delta(t)
\end{array}\right]
$$

For the sake of simplicity the Rabi frequences of the couplings between the ground states and the excited state $\Omega_{1}(t), \ldots, \Omega_{N}(t)$ are assumed real and positive as the populations do not depend on their signs. The phases of the couplings can easily be incorporated in the description and they can be used to control the inner phases of the created superposition states. Furthermore, the
Rabi frequencies are assumed to be pulse-shaped functions with the same time dependence $f(t)$, but possibly with different magnitudes,

$$
\Omega_{n}(t)=\chi_{n} f(t) \quad(n=1,2, \ldots, N),
$$

and hence different pulse areas,

$$
A_{n}=\int_{-\infty}^{\infty} \Omega_{n}(t) d t=\chi_{n} \int_{-\infty}^{\infty} f(t) d t \quad(n=1,2, \ldots, N) .
$$

\section{B. Physical implementations}

The linkage pattern described by the Hamiltonian (2) can be implemented experimentally in laser excitation of atoms or molecules. For example, the $N=3$ case is readily implemented in the $J=1 \leftrightarrow J=0$ system coupled by three laser fields with right circular, left circular and linear polarizations, as shown in Fig. 2 (left). These coupling fields can be produced from the same laser by standard optical tools (beam splitters, polarizers, etc.), which greatly facilitates implementation. Moreover, the use of pulses derived from the same laser ensures automatically the two-photon resonance conditions and the condition (3) for the same temporal profile of all pulses.

The cases of $N=4-6$ can be realized by adding an additional $J=1$ level to the coupling scheme and appropriately polarized laser pulses, as shown in the right frame of Fig. 2.
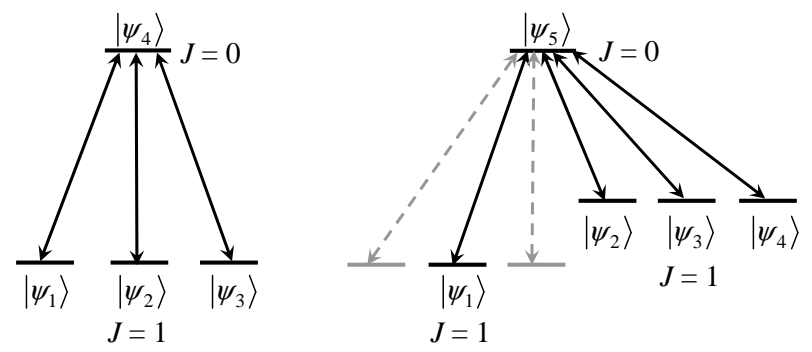

FIG. 2: Examples of physical implementations of the linkage pattern of $N$ degenerate ground states coupled via one upper state, considered in the present paper. Left: $N=3$ degenerate states. Right: $N=4$ degenerate (in the RWA sense) states (dashed arrows indicate two additional possible linkages).

\section{GENERAL SOLUTION}

\section{A. Morris-Shore (dark-bright) basis}

The Hamiltonian (2) has $N-1$ zero eigenvalues and two nonzero ones,

$$
\begin{aligned}
\lambda_{n} & =0 \quad(n=1, \ldots, N-1), \\
\lambda_{ \pm}(t) & =\frac{1}{2}\left[\Delta \pm \sqrt{\Delta^{2}+\Omega^{2}(t)}\right],
\end{aligned}
$$


where

$$
\Omega(t)=\sqrt{\sum_{n=1}^{N} \Omega_{n}^{2}(t)} \equiv \chi f(t)
$$

is the root-mean-square (rms) Rabi frequency, where

$$
\chi=\sqrt{\sum_{n=1}^{N} \chi_{n}^{2}}
$$

The set of orthonormalized eigenstates $\left|\varphi_{n}\right\rangle \quad(n=$ $1,2, \ldots, N-1)$ corresponding to the zero eigenvalues can be chosen as

$$
\begin{aligned}
\left|\varphi_{1}\right\rangle= & \frac{1}{X_{2}}\left[\chi_{2},-\chi_{1}, 0,0, \cdots, 0\right]^{T} \\
\left|\varphi_{2}\right\rangle= & \frac{1}{X_{2} X_{3}}\left[\chi_{1} \chi_{3}, \chi_{2} \chi_{3},-X_{2}^{2}, 0, \cdots, 0\right]^{T}, \\
\left|\varphi_{3}\right\rangle= & \frac{1}{X_{3} X_{4}}\left[\chi_{1} \chi_{4}, \chi_{2} \chi_{4}, \chi_{3} \chi_{4},-X_{3}^{2}, 0, \cdots, 0\right](8 \mathrm{~b}) \\
& \cdots \\
\left|\varphi_{N-1}\right\rangle= & \frac{1}{X_{N-1} X_{N}}\left[\chi_{1} \chi_{N}, \chi_{2} \chi_{N}, \cdots,-X_{N-1}^{2}, 0\right](8 \mathrm{~d})
\end{aligned}
$$

where

$$
X_{n}=\sqrt{\sum_{k=1}^{n} \chi_{k}^{2}} \quad(n=2,3, \ldots, N) .
$$

These eigenstates are dark states, i.e. they do not involve the excited state $\left|\psi_{N+1}\right\rangle$ and, as we shall see, are uncoupled from $\left|\psi_{N+1}\right\rangle$. All dark states are time-independent. We emphasize that the choice (8) of dark states is not unique because any superposition of dark states is a dark state too; hence their choice is a matter of convenience.

The Hilbert space is decomposed into two subspaces: an $(N-1)$-dimensional dark subspace comprising the dark states (8) and a two-dimensional subspace orthogonal to the dark subspace. It is convenient to use the Morris-Shore (MS) basis [11], which, in addition to the dark states, includes the excited state $\left|\psi_{N+1}\right\rangle \equiv\left|\varphi_{N+1}\right\rangle$ and a bright ground state $\left|\varphi_{N}\right\rangle$. The latter does not have a component of the excited state and is orthogonal to the dark states; these conditions determine it completely (up to an unimportant global phase),

$$
\left|\varphi_{N}\right\rangle=\frac{1}{X_{N}}\left[\chi_{1}, \chi_{2}, \cdots, \chi_{N}, 0\right]^{T} .
$$

We point out that the Morris-Shore basis is not the adiabatic basis because only the dark states are eigenstates of the Hamiltonian, but $\left|\varphi_{N}\right\rangle$ and $\left|\varphi_{N+1}\right\rangle$ are not.

In the new, still stationary basis $\left\{\left|\varphi_{n}\right\rangle\right\}_{n=1,2, \ldots, N+1}$, the Schrödinger equation reads

$$
i \hbar \frac{d}{d t} \mathbf{B}(t)=\widetilde{\mathrm{H}}(t) \mathbf{B}(t)
$$

where the original amplitudes $\mathbf{C}(t)$ are connected to the MS amplitudes $\mathbf{B}(t)$ by the time-independent unitary matrix $\mathrm{W}$ composed by the basis vectors $\left|\varphi_{n}\right\rangle$,

$$
\mathrm{W}=\left[\left|\varphi_{1}\right\rangle,\left|\varphi_{2}\right\rangle, \ldots,\left|\varphi_{N+1}\right\rangle\right]
$$

according to

$$
\mathbf{C}(t)=\mathbf{W B}(t)
$$

The transformed Hamiltonian reads $\widetilde{\mathrm{H}}(t)=\mathrm{W}^{\dagger} \mathrm{H}(t) \mathrm{W}$, or explicitly,

$$
\widetilde{\mathrm{H}}(t)=\frac{\hbar}{2}\left[\begin{array}{cccccc}
0 & 0 & \cdots & 0 & 0 & 0 \\
0 & 0 & \cdots & 0 & 0 & 0 \\
\vdots & \vdots & \ddots & \vdots & \vdots & \vdots \\
0 & 0 & \cdots & 0 & 0 & 0 \\
0 & 0 & \cdots & 0 & 0 & \Omega(t) \\
0 & 0 & \cdots & 0 & \Omega(t) & 2 \Delta(t)
\end{array}\right] .
$$

We point out that the Hamiltonian of Eq. (2) is a special case of the most general Hamiltonian for which the MS transformation [11] applies and which includes $N$ degenerate lower states and $M$ degenerate upper states. Hamiltonians of the same type as (2) and related transformations leading to Eq. (14), have appeared in the literature also before the paper by Morris and Shore [11], mostly in simplified versions of constant and equal interactions (see e.g. [16] and references therein).

\section{B. Solution in the Morris-Shore basis}

As evident from the first $N-1$ zero rows of $\widetilde{\mathrm{H}}$ the dark states are decoupled from states $\left|\varphi_{N}\right\rangle$ and $\left|\varphi_{N+1}\right\rangle$ and the dark-state amplitudes remain unchanged, $B_{n}(t)=$ const $(n=1,2, \ldots, N-1)$. Thus the $(N+1)$-state problem reduces to a two-state one involving $\left|\varphi_{N}\right\rangle$ and $\left|\varphi_{N+1}\right\rangle$,

$$
i \frac{d}{d t}\left[\begin{array}{c}
B_{N} \\
B_{N+1}
\end{array}\right]=\frac{1}{2}\left[\begin{array}{cc}
0 & \Omega \\
\Omega & 2 \Delta
\end{array}\right]\left[\begin{array}{c}
B_{N} \\
B_{N+1}
\end{array}\right] .
$$

The propagator for this two-state system, defined by

$$
\left[\begin{array}{c}
B_{N}(+\infty) \\
B_{N+1}(+\infty)
\end{array}\right]=\mathrm{U}_{M S}^{(2)}\left[\begin{array}{c}
B_{N}(-\infty) \\
B_{N+1}(-\infty)
\end{array}\right]
$$

is unitary and can be expressed in terms of the CayleyKlein parameters as

$$
\mathrm{U}_{M S}^{(2)}=\left[\begin{array}{cc}
a & b \\
-b^{*} & a^{*}
\end{array}\right],
$$

with $|b|^{2}=1-|a|^{2}$. Then the transition matrix for the $(N+1)$-state system in the MS basis reads

$$
\mathrm{U}_{M S}^{(N+1)}=\left[\begin{array}{cccccc}
1 & 0 & \cdots & 0 & 0 & 0 \\
0 & 1 & \cdots & 0 & 0 & 0 \\
\vdots & \vdots & \ddots & \vdots & \vdots & \vdots \\
0 & 0 & \cdots & 1 & 0 & 0 \\
0 & 0 & \cdots & 0 & a & b \\
0 & 0 & \cdots & 0 & -b^{*} & a^{*}
\end{array}\right] .
$$




\section{The solution in the original basis}

We can find the transition matrix in the original, diabatic basis by using the transformation

$$
\mathrm{U}^{(N+1)}(\infty,-\infty)=\mathrm{WU}_{M S}^{(N+1)}(\infty,-\infty) \mathrm{W}^{\dagger}
$$

or explicitly,

$$
\mathbf{U}^{(N+1)}=\left[\begin{array}{cccccc}
1+(a-1) \frac{\chi_{1}^{2}}{\chi^{2}} & (a-1) \frac{\chi_{1} \chi_{2}}{\chi^{2}} & (a-1) \frac{\chi_{1} \chi_{3}}{\chi^{2}} & \cdots & (a-1) \frac{\chi_{1} \chi_{N}}{\chi^{2}} & b \frac{\chi_{1}}{\chi} \\
(a-1) \frac{\chi_{1} \chi_{2}}{\chi^{2}} & 1+(a-1) \frac{\chi_{2}^{2}}{\chi^{2}} & (a-1) \frac{\chi_{2} \chi_{3}}{\chi^{2}} & \cdots & (a-1) \frac{\chi_{2} \chi_{N}}{\chi^{2}} & b \frac{\chi_{2}}{\chi} \\
(a-1) \frac{\chi_{1} \chi_{3}}{\chi^{2}} & (a-1) \frac{\chi_{2} \chi_{3}}{\chi^{2}} & 1+(a-1) \frac{\chi_{3}^{2}}{\chi^{2}} & \cdots & (a-1) \frac{\chi_{3} \chi_{N}}{\chi^{2}} & b \frac{\chi_{3}}{\chi} \\
\vdots & \vdots & \vdots & \ddots & \vdots & \vdots \\
(a-1) \frac{\chi_{1} \chi_{N}}{\chi^{2}} & (a-1) \frac{\chi_{2} \chi_{N}}{\chi^{2}} & (a-1) \frac{\chi_{3} \chi_{N}}{\chi^{2}} & \cdots & 1+(a-1) \frac{\chi_{N}^{2}}{\chi^{2}} & b \frac{\chi_{N}}{\chi} \\
-b^{*} \frac{\chi_{1}}{\chi} & -b^{*} \frac{\chi_{2}}{\chi} & -b^{*} \frac{\chi_{3}}{\chi} & \cdots & -b^{*} \frac{\chi_{N}}{\chi} & a^{*}
\end{array}\right]
$$

The $i$ th column of this matrix provides the probability amplitudes for initial conditions

$$
\begin{aligned}
C_{i}(-\infty) & =1, \\
C_{n}(-\infty) & =0 \quad(n \neq i) .
\end{aligned}
$$

The initial state $\left|\psi_{i}\right\rangle$ can be one of the degenerate states or the upper state. This general unitary matrix and combinations of such matrices can be used to design techniques for general or special qunit rotations.

As evident from Eq. (20) for finding the populations for the initial condition (21) it is sufficient to know only the parameter $a=\left[U_{M S}^{(2)}(\infty,-\infty)\right]_{11}$ because $|b|^{2}=1-$ $|a|^{2}$ [17]. For the sake of simplicity, in the present paper we are interested only in cases when the system starts in a single state and below we shall concentrate on the values of the parameter $a$. In the more general case when the system starts in a coherent superposition of states, Eq. (20) can be used again to derive the solution; then the other Cayley-Klein parameter $b$ is also needed.

\section{TYPES OF POPULATION DISTRIBUTION}

We identify two types of initial conditions: when the system starts in one of the degenerate states $\left|\psi_{i}\right\rangle$ or in the excited state $\left|\psi_{N+1}\right\rangle$, which we shall consider separately.

\section{A. System initially in a ground state}

\section{General case}

When the system is initially in the ground state $\left|\psi_{i}\right\rangle$, Eq. (21), we find from the $i$ th column of the propagator
(20) that the populations in the end of the evolution are

$$
\begin{aligned}
P_{i} & =\left|1+(a-1) \frac{\chi_{i}^{2}}{\chi^{2}}\right|^{2}, \\
P_{n} & =|a-1|^{2} \frac{\chi_{i}^{2} \chi_{n}^{2}}{\chi^{4}} \quad(n \neq i, N+1), \\
P_{N+1} & =\left(1-|a|^{2}\right) \frac{\chi_{i}^{2}}{\chi^{2}}
\end{aligned}
$$

Therefore the ratio of the populations of any two degenerate states, different from the initial state $\left|\psi_{i}\right\rangle$, reads

$$
\frac{P_{m}}{P_{n}}=\frac{\chi_{m}^{2}}{\chi_{n}^{2}} \quad(m, n \neq i, N+1) .
$$

Hence these population ratios do not depend on the interaction details but only on the ratios of the corresponding peak Rabi frequencies.

For equal Rabi frequencies,

$$
\chi_{1}=\chi_{2}=\cdots=\chi_{N},
$$

Eqs. (22) reduce to

$$
\begin{aligned}
P_{i} & =\left|1+\frac{a-1}{N}\right|^{2}, \\
P_{n} & =\frac{|a-1|^{2}}{N^{2}} \quad(n \neq i, N+1), \\
P_{N+1} & =\frac{1-|a|^{2}}{N} .
\end{aligned}
$$

Thus the populations of all ground states except the initial state $\left|\psi_{i}\right\rangle$ are equal. 


\section{Special values of a}

Several values of the propagator parameter $a$ are especially interesting.

For $a=0$, which indicates complete population transfer (CPT) in the MS two-state system, Eq. (22) gives

$$
\begin{aligned}
P_{i} & =\left|1-\frac{\chi_{i}^{2}}{\chi^{2}}\right|^{2} \\
P_{n} & =\frac{\chi_{n}^{2} \chi_{i}^{2}}{\chi^{4}} \quad(n \neq i, N+1) \\
P_{N+1} & =\frac{\chi_{i}^{2}}{\chi^{2}}
\end{aligned}
$$

For $a=1$, which corresponds to complete population return $(\mathrm{CPR})$ in the $\mathrm{MS}$ two-state system, we obtain

$$
\begin{aligned}
P_{i} & =1, \\
P_{n} & =0 \quad(n \neq i, N+1), \\
P_{N+1} & =0 .
\end{aligned}
$$

For $a=-1$, which again corresponds to CPR in the MS two-state system, but with a sign flip in the amplitude, we find

$$
\begin{aligned}
P_{i} & =\left(1-2 \frac{\chi_{i}^{2}}{\chi^{2}}\right)^{2}, \\
P_{n} & =\frac{4 \chi_{n}^{2} \chi_{i}^{2}}{\chi^{4}} \quad(n \neq i, N+1), \\
P_{N+1} & =0 .
\end{aligned}
$$

It is important to note that although both cases $a=1$ and $a=-1$ lead to CPR in the MS two-state system, they produce very different population distributions in the full $(N+1)$-state system. The case $a=1$ leads to a trivial result (CPR in the full system), whereas the case $a=-1$ is very interesting because it leads to a population redistribution amongst the ground states with zero population in the upper state; hence this case deserves a special attention.

\section{The case $a=-1$}

The case of $a=-1$ is particularly important because it allows to create a coherent superposition of all ground states, with no population in the upper state.

All ground-state populations in this superposition will be equal,

$$
\begin{aligned}
& P_{1}=P_{2}=\cdots=P_{N}=\frac{1}{N}, \\
& P_{N+1}=0 .
\end{aligned}
$$

if

$$
\begin{gathered}
\chi_{i}=(\sqrt{N} \pm 1) \chi_{0}, \\
\chi_{n}=\chi_{0} \quad(n \neq i),
\end{gathered}
$$

where

$$
\chi_{0}=\frac{\chi}{\sqrt{2(N \pm \sqrt{N})}}
$$

This result does not depend on other interaction details (pulse shape, pulse area, detuning) as long as $a=-1$. For example, for $N=4$ degenerate states, equal populations are obtained when $\chi_{i}=\chi_{n}$ or $\chi_{i}=3 \chi_{n}$. We shall discuss later how the condition $a=-1$ can be obtained for several analytically soluble models.

Another important particular case is when the initialstate population $P_{i}$ vanishes in the end. This occurs for

$$
\chi_{i}^{2}=\sum_{n \neq i} \chi_{n}^{2} .
$$

For example, an equal superposition of all lower sublevels except $\left|\psi_{i}\right\rangle$,

$$
\begin{aligned}
P_{i} & =P_{N+1}=0, \\
P_{n} & =\frac{1}{N-1} \quad(n \neq i, N+1),
\end{aligned}
$$

is created for

$$
\begin{aligned}
\chi_{i} & =\chi_{0} \sqrt{N-1}, \\
\chi_{n} & =\chi_{0} \quad(n \neq i),
\end{aligned}
$$

where

$$
\chi_{0}=\frac{\chi}{\sqrt{2(N-1)}} .
$$

\section{B. System initially in the upper state}

If the system is initially in the excited state $\left|\psi_{N+1}\right\rangle$, at the end of the evolution the populations will be

$$
\begin{aligned}
& P_{n}=\left(1-|a|^{2}\right) \frac{\chi_{n}^{2}}{\chi^{2}} \quad(n=1,2, \ldots, N), \\
& P_{N+1}=|a|^{2} .
\end{aligned}
$$

For $a= \pm 1$ at the end of the evolution the system undergoes CPR, as in the MS two-state system. For $a=0$ (CPT in the MS two-state system) the whole population will be in the ground states leaving the excited state empty, $P_{N+1}=0$. If all the couplings are equal, Eq. (24), the ground states will have equal populations,

$$
P_{n}=\frac{1}{N} \quad(n=1,2, \ldots, N) .
$$




\section{Discussion}

In this section we discussed some general features of the population redistribution in the $(N+1)$-state system. There are three particularly interesing results.

First, the ratios of the populations of the degenerate states (except the one populated initially) depend only on the ratios of the corresponding Rabi frequencies; hence they can be controlled by changing the corresponding laser intensities alone. The populations values, though, depend on the other interaction details. Moreover, it can easily be seen that the relative phases of the degenerate states can be controlled by the relative laser phases.

Second, it is possible to create an equal superposition of all ground states, with zero population in the upper state. This is possible when the system starts in a ground state: then condition (30) is required, along with the CPR condition $a=-1$. Alternatively, an equal superposition can be created when the system starts in the upper state: then condition (24) is required, along with the CPT condition $a=0$. Equal superpositions are important in some applications because they are states with maximal coherence (since the population inversions vanish).

Third, it is possible, starting from a ground state, to create a superposition of all other ground states, whereas the initial ground state and the excited state are left unpopulated. This requires $a=-1$ and condition (31). This case has interesting physical implications, which will be discussed in the next section.

\section{APPLICATIONS TO EXACTLY SOLUBLE MODELS}

\section{A. Multistate analytical solutions}

The values of the propagator parameter $a=$ $\left[U_{M S}^{(2)}(\infty,-\infty)\right]_{11}$ for the most popular analytically exactly soluble models are listed in Table I Equation (20), supplied with these values, provides several exact multistate analytical solutions, which generalize the respective two-state solutions.

Among these solutions, the resonance case is the simplest and most important one, which will receive a special attention below. It will be followed by a detailed discussion of the Rosen-Zener (RZ) model, which can be seen as an extension of the resonance solution to nonzero detuning for a special pulse shape (hyperbolic secant). Both the resonance and the $\mathrm{RZ}$ model allow for the parameter $a$ to obtain the important values $0, \pm 1$. The Rabi model can also be used to illustrate the interesting cases of population distribution associated with these values of $a$ but its rectangular pulse shape is less attractive (and also less realistic) than the beautiful sech-shape of the pulse in the RZ model.

The Landau-Zener (LZ) and Allen-Eberly (AE) models are of level-crossing type, i.e. the detuning crosses

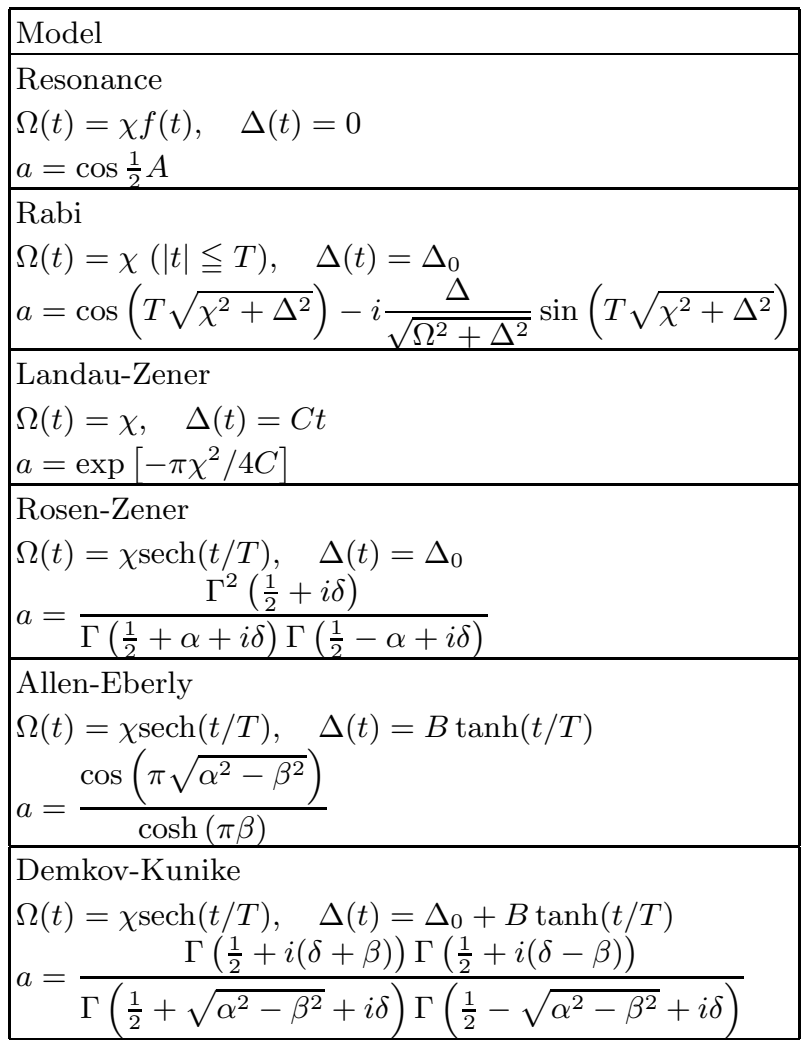

TABLE I: Values of the Cayley-Klein parameter $a=$ $\left[U_{M S}^{(2)}(\infty,-\infty)\right]_{11}$ for several exactly soluble models. Here $\Gamma(z)$ is the Gamma function and $\alpha=\frac{1}{2} \chi T, \beta=\frac{1}{2} B T$, $\delta=\frac{1}{2} \Delta_{0} T$, are scaled dimensionless parameters, which are assumed positive without loss of generality.

resonance, $\Delta(0)=0$. For these models in the adiabatic limit the transition probability approaches unity, that is $a \rightarrow 0$. The parameter $a$ is always nonnegative, i.e. the most interesting value in the present context, $a=-1$, is unreachable. Nevertheless, because of the popularity and the importance of the LZ model, and because the present multistate LZ solution supplements other multistate LZ solutions, we discuss this solution in detail in Sec. VI.

The Demkov-Kunike (DK) model is a very versatile model, which combines and generalizes the RZ and AE models. Indeed, as seen in Table I, the DK model reduces to the RZ model for $B=0$ and to the AE model for $\Delta_{0}=0$. For the DK model, the parameter $a$ can be equal to the most interesting value of -1 only when $B=0$, i.e. only in the RZ limit. Therefore, we shall only consider the RZ model below, and leave the AE and DK models to readers interested in other aspects of the analytic multistate solutions presented here.

\section{B. Exact resonance}

In the case of exact resonance,

$$
\Delta=0
$$


the elements of the evolution matrix for the MS two-state system for any pulse shape of $\Omega(t)$ are

$$
\begin{aligned}
& a=\cos \frac{A}{2}, \\
& b=-i \sin \frac{A}{2},
\end{aligned}
$$

where $A$ is the rms pulse area defined as

$$
A=\int_{-\infty}^{\infty} \Omega\left(t^{\prime}\right) d t^{\prime}
$$

In the important case of $N=3$ we have

$$
\mathrm{U}_{d}^{(4)}=\left[\begin{array}{ccccc}
1-2 \frac{\chi_{1}^{2}}{\chi^{2}} \sin ^{2} \frac{1}{4} A & -2 \frac{\chi_{1} \chi_{2}}{\chi^{2}} \sin ^{2} \frac{1}{4} A & -2 \frac{\chi_{1} \chi_{3}}{\chi^{2}} \sin ^{2} \frac{1}{4} A & -i \frac{\chi_{1}}{\chi} \sin \frac{1}{2} A \\
-2 \frac{\chi_{1} \chi_{2}}{\chi^{2}} \sin ^{2} \frac{1}{4} A & 1-2 \frac{\chi_{2}^{2}}{\chi^{2}} \sin ^{2} \frac{1}{4} A & -2 \frac{\chi_{2} \chi_{3}}{\chi^{2}} \sin ^{2} \frac{1}{4} A & -i \frac{\chi_{2}}{\chi} \sin \frac{1}{2} A \\
-2 \frac{\chi_{1} \chi_{3}}{\chi^{2}} \sin ^{2} \frac{1}{4} A & -2 \frac{\chi_{2} \chi_{3}}{\chi^{2}} \sin ^{2} \frac{1}{4} A & 1-2 \frac{\chi_{3}^{2}}{\chi^{2}} \sin ^{2} \frac{1}{4} A & -i \frac{\chi_{3}}{\chi} \sin \frac{1}{2} A \\
-i \frac{\chi_{1}}{\chi} \sin \frac{1}{2} A & -i \frac{\chi_{2}}{\chi} \sin \frac{1}{2} A & -i \frac{\chi_{3}}{\chi} \sin \frac{1}{2} A & \cos \frac{1}{2} A
\end{array}\right]
$$

We have $a=0, \pm 1$ for the following pulse areas,

$$
\begin{aligned}
a=0: & A=(2 l+1) \pi, \\
a=1: & A=4 l \pi, \\
a=-1: & A=2(2 l+1) \pi .
\end{aligned}
$$

where $l=0,1,2, \ldots$.

The pulse areas for the three important cases discussed in Sec. IV C are easily calculated.

An equal superposition of all $N$ ground states is created when starting from the excited state and all individual pulse areas are equal to (see Sec. IV B)

$$
A_{n}=\frac{(2 l+1) \pi}{\sqrt{N}} \quad(n=1,2, \ldots, N),
$$

where $l=0,1,2, \ldots$.

An equal superposition of all $N$ ground states is created also when starting from one ground state $\left|\psi_{i}\right\rangle$ and the pulse areas are [see Eq. (30)]

$$
\begin{aligned}
& A_{i}=\sqrt{2 \frac{\sqrt{N} \pm 1}{\sqrt{N}}}(2 l+1) \pi, \\
& A_{n}=\sqrt{\frac{2}{N \pm \sqrt{N}}}(2 l+1) \pi \quad(n \neq i),
\end{aligned}
$$

where $l=0,1,2, \ldots$

The other interesting case when the system starts in one ground state $\left|\psi_{i}\right\rangle$ and ends up in an equal superposition of all other ground states is realised for pulse areas [see Eq. (33)]

$$
\begin{aligned}
& A_{i}=\sqrt{2}(2 l+1) \pi \\
& A_{n}=\sqrt{\frac{2}{N-1}}(2 l+1) \pi \quad(n \neq i),
\end{aligned}
$$

where $l=0,1,2, \ldots$

\section{Multistate Rosen-Zener model}

Equation (20) and the value of the parameter $a$ in Table I represent the multistate RZ solution in the degenerate two-level system. It is easy to show that

$$
|a|^{2}=1-\frac{\sin ^{2}\left(\frac{1}{2} \pi \chi T\right)}{\cosh ^{2}\left(\frac{1}{2} \pi \Delta_{0} T\right)},
$$

where we have used the reflection formula $\Gamma\left(\frac{1}{2}+z\right) \Gamma\left(\frac{1}{2}-\right.$ $z)=\pi / \cos \pi z$ [18]. Hence in this model $|a|=1$ for $\alpha=\frac{1}{2} \chi T=l(l=0,1,2, \ldots)$. The phase of $a$, however, depends on the detuning $\Delta_{0}$ [17]; we use this to an advantage to select values of $\Delta_{0}$ for which $a=-1$. For $\alpha=l$ we find 17

$$
a=(-1)^{n} \prod_{k=0}^{n-1} \frac{2 l+1-i \Delta_{0} T}{2 l+1+i \Delta_{0} T} \quad(\alpha=l),
$$

where the recurrence relation $\Gamma(z+1)=z \Gamma(z)$ [18] has been used. Thus, the equation $a=-1$ reduces to an algebraic equation for $\Delta_{0}$, which has $l$ real solutions [17]. The first few values of $\chi$ and $\Delta_{0}$ for which $a=-1$ are shown in Table [I] As the table shows, $\Delta_{0}=0$ is a solution for odd $\alpha=\frac{1}{2} \chi T$ but not for even $\alpha$, in agreement with the conclusions in Sec. VB. Moreover, the $a=-1$ solutions do not depend on the number of degenerate states $N$.

In the present context the RZ model is interesting for it shows that one can create superpositions within the ground-state manifold even when the excited state is off resonance by a considerable detuning $\left(\Delta_{0} \gg 1 / T\right)$, for which the transition probability in the MS two-state system is virtually zero, i.e. $|a| \approx 1$. This fact allows us, for specific detunings, to essentially contain the transient dynamics within the ground states; in contrast, in the resonance case the excited state can get significant transient population, $P_{N+1}(t)=\sin ^{2} \frac{1}{2} A(t)$, although it vanishes in the end.

Figure 3 displays the populations against the detuning $\Delta_{0}$ for a hyperbolic-secant pulse with $\chi T=18$ for 


\begin{tabular}{|r|rrl|}
\hline$\chi T$ & $\Delta_{0} T$ & & \\
\hline 2 & 0 & & \\
4 & \pm 1.732 & & \\
6 & 0 & \pm 4.796 & \\
8 & \pm 1.113 & \pm 9.207 & \\
10 & 0 & \pm 2.756 & \pm 14.913 \\
12 & \pm 0.943 & \pm 4.936 & \pm 21.903 \\
14 & 0 & \pm 2.243 & $\pm 7.595 \pm 30.171$ \\
16 & \pm 0.855 & \pm 3.916 & $\pm 10.708 \pm 39.715$ \\
18 & 0 & \pm 1.988 & $\pm 5.907 \pm 14.265 \pm 50.534$ \\
20 & \pm 0.799 & \pm 3.418 & $\pm 8.195 \pm 18.260 \pm 62.627$ \\
22 & 0 & \pm 1.830 & $\pm 5.098 \pm 10.766 \pm 22.687 \pm 75.993$ \\
24 & \pm 0.759 & \pm 3.113 & $\pm 7.006 \pm 13.613 \pm 27.545 \pm 90.634$ \\
26 & 0 & \pm 1.719 & $\pm 4.606 \quad \pm 9.130 \pm 16.729 \pm 32.833$ \\
& & \pm 106.549 & \\
28 & \pm 0.728 & \pm 2.901 & $\pm 6.289 \pm 11.461 \pm 20.113 \pm 38.548$ \\
& \multicolumn{5}{|c|}{ \pm 123.736} \\
30 & 0 & \pm 1.636 & $\pm 4.268 \quad \pm 8.150 \pm 13.994 \pm 23.760$ \\
& \multicolumn{5}{|c|}{ $\pm 44.690 \pm 142.198$} \\
\hline
\end{tabular}

TABLE II: Some approximate solutions of the equation $a\left(\Delta_{0}\right)=-1$ for the RZ model, where $a$ is given in Table [1. for various even integer values of $\chi T$.

couplings chosen to satisfy Eqs. (30) (upper frame) and (33) (lower frame). In both cases we have $|a|=1$ [see Eq. [44)], which leaves the excited state unpopulated in the end. For several special values of the detuning $\Delta_{0}$, as predicted in Table II, we have $a=-1$. For these values, an equal superposition of all degenerate states including the initially populated state $\left|\psi_{1}\right\rangle$ is created in the upper frame, and an equal superposition of all degenerate states except $\left|\psi_{1}\right\rangle$ is created in the lower frame.

Figure 4 shows the final populations versus the rms pulse area $A=\pi \chi T$ for $N=3$ degenerate lower states for couplings chosen to satisfy Eqs. (30) (upper frame) and (33) (lower frame). As follows from Table [I] an equal superposition of all degenerate states is created for rms pulse area $A=18 \pi$; this is indeed seen in the figure in the upper frame. For the same value of $A$ in the lower frame an equal superposition is created of all degenerate states except the initially populated state $\left|\psi_{1}\right\rangle$. In both frames, there are other values of $A$ for which the same superpositions are apparently created; a closer examination (not shown) reveals that for these other values of the rms pulse area the created superposition has almost, but not exactly, equal components.

Figure 5 displays the time evolution of the populations for $N=3$ degenerate lower states and rms pulse area of $18 \pi$, and two detunings: $\Delta=0$ in the upper frame and $\Delta T=50.534$ in the lower frame. For these pairs of areas and detunings, Figs. 3 and 4 have already demonstrated that an equal superposition of all degenerate states is created. Figure 5 shows that the evolution towards such a superposition can be dramatically different on and off

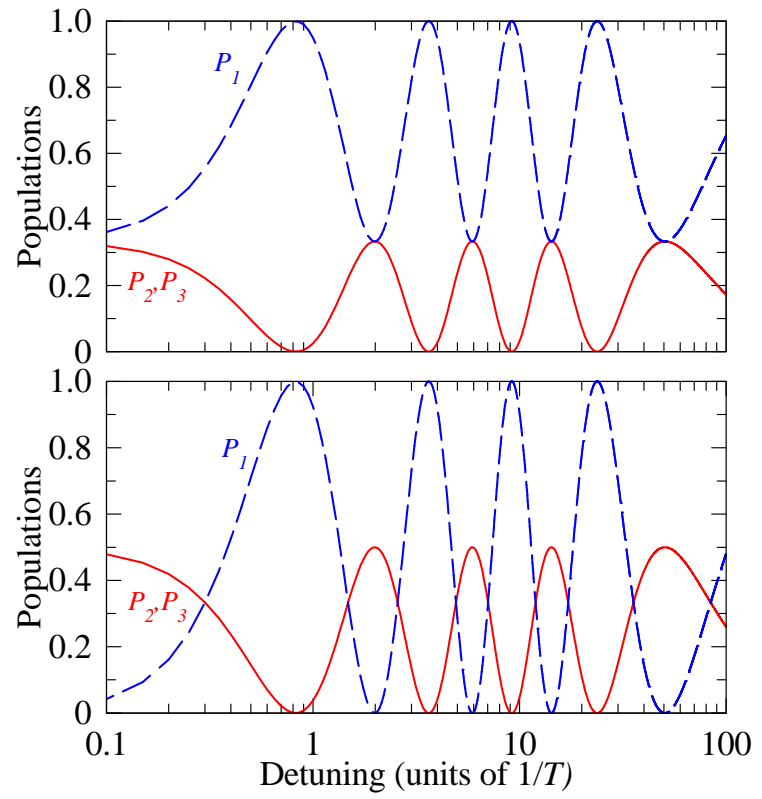

FIG. 3: (Color online) Populations vs the detuning $\Delta_{0}$ for $N=3$ lower states and $\chi T=18$. The coupling strengths $\chi_{n}$ are given by Eqs. (30) in the upper frame and Eqs. (33) in the lower frame. The system is initially in state $\left|\psi_{1}\right\rangle$.

resonance. Indeed, for $\Delta=0$ (upper frame) the nondegenerate upper state receives considerable transient population, which would lead to significant losses if this state can decay on the time scale of the pulsed interaction. In strong contrast, off resonance this undesired population is greatly reduced (lower frame), and still the desired equal superposition of the degenerate states emerges in the end. We have verified numerically that for larger detunings this transient population continues to decrease, e.g. for $\Delta T=142.198$ and $\Omega T=30$ it is less than $1 \%$.

To conclude this section we point out that one can create any desired superposition, with arbitrary unequal populations, in very much the same manner, on or off resonance, by appropriately chosing the individual couplings, while still maintaning particular values of the overall rms pulse area. Tuning on resonance gives the advantage of smaller pulse area required, whereas tuning off resonance (with larger pulse area) provides the advantage of greatly reducing the transient population of the possibly lossy common upper state.

\section{MULTISTATE LANDAU-ZENER MODEL}

As seen in Table $\prod$ the propagator parameter $a$ for the LZ model, $a=\exp \left(-\pi \chi^{2} / 4 C\right)$, cannot be equal to 0 or 1 or -1 , but may approach 0 or 1 arbitrarily closely. However, it is always positive and cannot approach the value of -1 ; hence the LZ model is unsuitable for unitary operations within the degenerate manifold, in contrast to the resonance and RZ models discussed above. Still, the present multistate LZ solution represents an interesting 

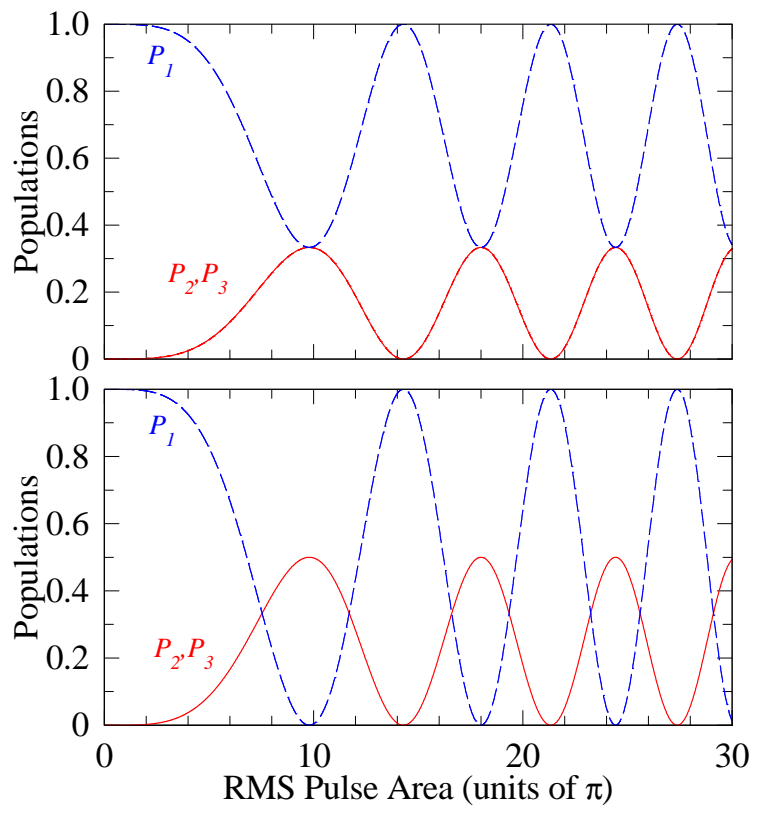

FIG. 4: (Color online) Final populations versus the rms pulse area $\chi$ for $N=3$ degenerate lower states and detuning $\Delta T=$ 50.534. The coupling strengths $\chi_{n}$ are given by Eqs. (30) in the upper frame and Eqs. (33) in the lower frame. The system is initially in state $\left|\psi_{1}\right\rangle$.

and important addition to the available LZ solutions (see [19] and references therein).

\section{The Demkov-Osherov model}

The present multistate LZ model complements the Demkov-Osherov (DO) model [20], wherein a slanted energy crosses $N$ parallel nondegenerate energies. In the DO model, the exact probabilities $P_{n \rightarrow m}$ have the same form - products of LZ probabilities for transition or notransition applied at the relevant crossings - as what would be obtained by naive multiplication of LZ probabilities while moving across the grid of crossings from $\left|\psi_{n}\right\rangle$ to $\left|\psi_{m}\right\rangle$, without accounting for phases and interferences. For example, if the states $\left|\psi_{n}\right\rangle(n=1,2, \ldots, N)$ are labeled such that their energies increase with the index $n$, and if the slope of the slanted energy of state $\left|\psi_{N+1}\right\rangle$ is positive, the transition probabilities in the DO model are

$$
\begin{array}{rlr}
P_{n \rightarrow m} & =p_{n} q_{n+1} q_{n+2} \cdots q_{m-1} p_{m} & (n<m(46 \mathrm{a}) \\
P_{n \rightarrow m} & =0 \quad(n>m), & (46 \mathrm{~b}) \\
P_{n \rightarrow n} & =q_{n}, & (46 \mathrm{c}) \\
P_{n \rightarrow N+1} & =p_{n} q_{n+1} q_{n+2} \cdots q_{N}, & (46 \mathrm{~d}) \\
P_{N+1 \rightarrow n} & =q_{1} q_{2} \cdots q_{n-1} p_{n}, \\
P_{N+1 \rightarrow N+1} & =q_{1} q_{2} \cdots q_{N},
\end{array}
$$

where $q_{n}=\exp \left(-\pi \chi_{n}^{2} / 2 C\right)$ is the no-transition probability and $p_{n}=1-q_{n}$ is the transition probability between
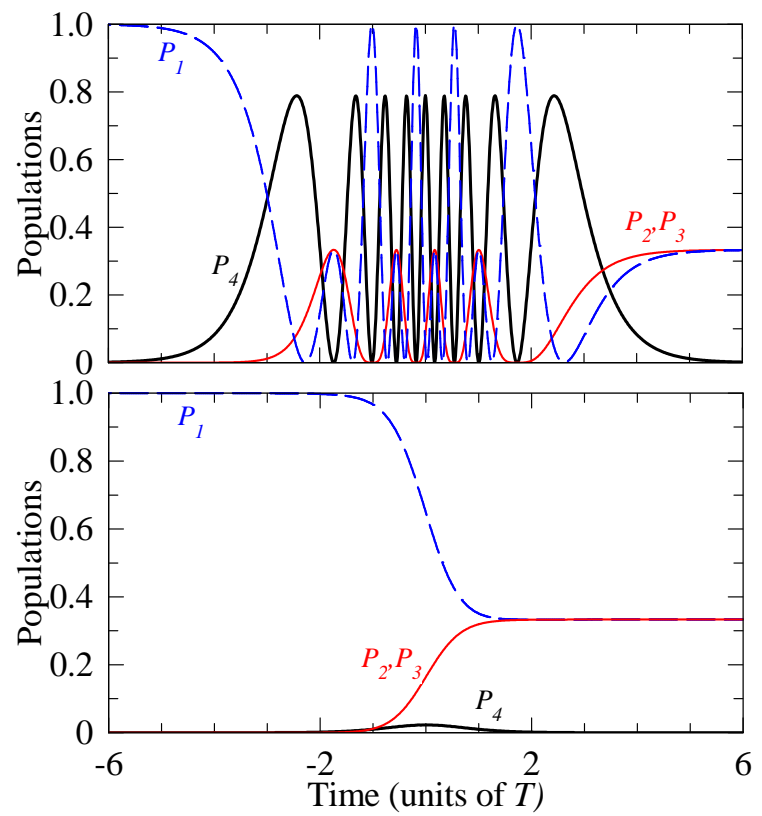

FIG. 5: (Color online) Populations versus time for $N=3$ lower states and rms Rabi frequency $\chi T=18$. The coupling strengths $\chi_{n}$ are given by Eqs. (30). The detuning is $\Delta=0$ in the upper frame and $\Delta T=50.534$ in the lower frame. The system is initially in state $\left|\psi_{1}\right\rangle$.

states $\left|\psi_{N+1}\right\rangle$ and $\left|\psi_{n}\right\rangle$ at the crossing of their energies.

\section{The degenerate case}

The present multistate LZ solution provides the transition probabilities for the special case when all parallel energies are degenerate, which cannot be obtained from the DO model.

a. The propagator The elements of the transition matrix for our $(N+1)$-state degenerate LZ problem are readily found from Eq. (20) to be

$$
\begin{array}{r}
U_{m, n}=-\frac{\chi_{n} \chi_{m}}{\chi^{2}}\left(1-e^{-\Lambda}\right) \quad(m, n=1, \ldots, N ; m \neq n), \\
U_{n, n}=1-\frac{\chi_{n}^{2}}{\chi^{2}}\left(1-e^{-\Lambda}\right) \quad(n=1, \ldots, N), \quad(47 \mathrm{a}) \\
U_{n, N+1}=\frac{\chi_{n}}{\chi} b \quad(n=1, \ldots, N), \\
U_{N+1, n}=-\frac{\chi_{n}}{\chi} b^{*} \quad(n=1, \ldots, N), \\
U_{N+1, N+1}=e^{-\Lambda},
\end{array}
$$

with $\Lambda=\pi \chi^{2} / 4 C$ and $|b|^{2}=1-e^{-2 \Lambda}$.

$b$. System initially in the nondegenerate state When the system begins initially in the nondegenerate state $\left|\psi_{N+1}\right\rangle$, with the tilted energy, the system ends in a co- 
herent superposition of all states with populations

$$
\begin{aligned}
P_{n} & =\frac{\chi_{n}^{2}}{\chi^{2}}\left(1-e^{-2 \Lambda}\right) \quad(n=1, \ldots, N), \\
P_{N+1} & =e^{-2 \Lambda} .
\end{aligned}
$$

In the adiabatic limit $\Lambda \gg 1$ the population is distributed among the degenerate states according to their couplings, whereas the initially populated state $\left|\psi_{N+1}\right\rangle$ is almost depleted, $P_{N+1} \approx 0$. For equal couplings, all degeneratestate populations will be equal, $P_{n} \approx 1 / N$. In the opposite, diabatic limit $\Lambda \ll 1$ the population remains in state $\left|\psi_{N+1}\right\rangle$ with almost no population in the degenerate states.

c. System initially in a degenerate state When the system is initially in an arbitrary degenerate state $\left|\psi_{i}\right\rangle$, at the end of the evolution the populations are

$$
\begin{aligned}
P_{i} & =\left[1-\frac{\chi_{i}^{2}}{\chi^{2}}\left(1-e^{-\Lambda}\right)\right]^{2} \\
P_{n} & =\frac{\chi_{n}^{2} \chi_{i}^{2}}{\chi^{4}}\left(1-e^{-\Lambda}\right)^{2} \quad(n=1, \ldots, N ; n \neq(x) \\
P_{N+1} & =\frac{\chi_{i}^{2}}{\chi^{2}}\left(1-e^{-2 \Lambda}\right) .
\end{aligned}
$$

In the adiabatic limit $\Lambda \gg 1$ and for equal couplings, the populations will be

$$
\begin{aligned}
P_{i} & \approx\left(1-\frac{1}{N}\right)^{2}, \\
P_{n} & \approx \frac{1}{N^{2}} \quad(n=1, \ldots, N ; n \neq i), \\
P_{N+1} & \approx \frac{1}{N} .
\end{aligned}
$$

Obviously, Eqs. (48) and (49) cannot be reduced to the DO solution (46), which implies that the non-degeneracy assumption in the DO model is essential.

Figure [6 shows the transition probability for the multistate LZ model plotted against the LZ parameter $\Lambda=$ $\pi \chi^{2} / 4 C$. As $\Lambda$ increases the populations approach their steady adiabatic values (50). Different coherent superpositions can be created by choosing appropriate values for the couplings $\chi_{n}$.

\section{CONCLUSIONS}

In this paper we have described a procedure for deriving analytical solutions for a multistate system composed of $N$ degenerate lower states coupled via a nondegenerate upper state with pulsed interactions of the same temporal dependence but possibly with different peak amplitudes. The multistate resonance and Rosen-Zener solutions have been discussed in some detail because they allow one to find special values of parameters, termed

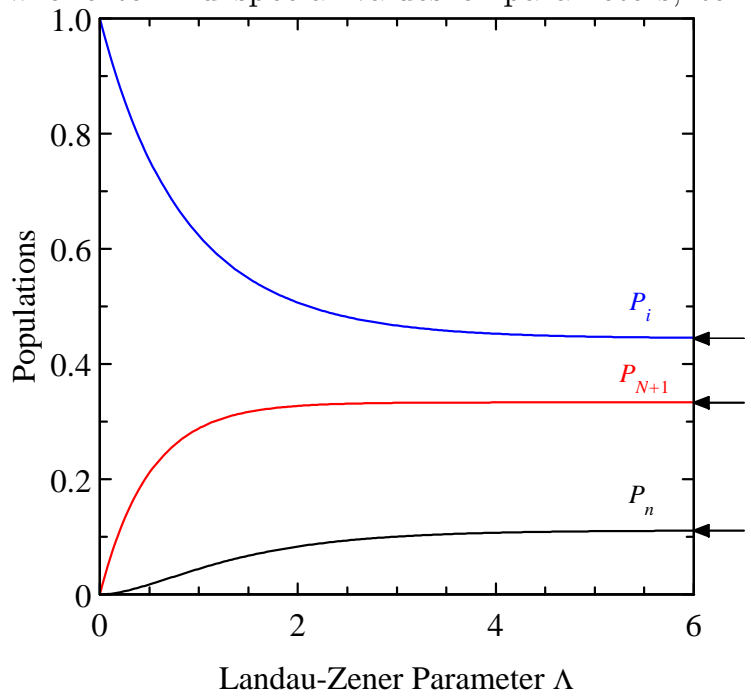

FIG. 6: (Color online) Populations for the degenerate LZ model vs the LZ parameter $\Lambda=\pi \chi^{2} / 4 C$ for $N=3$ degenerate states and equal couplings. The system is supposed to start in one of the degenerate states $\left|\psi_{i}\right\rangle$. The arrows on the right point the adiabatic values (50).

generalised $\pi$ pulses, for which various types of population transfer can occur, for example, creation of maximally coherent superpositions. The RZ solution is particularly useful because it allows to prescribe appropriately detuned pulsed fields for which the dynamics can be essentially contained within the degenerate-state space, without populating the upper state even transiently, thus avoiding possible losses from this state via spontaneous emission, ionization, etc.

We have analyzed in some detail also the multistate Landau-Zener model, which complements the DemkovOsherov model in the case of degenerate energies.

The presented analytical solutions and general properties have a significant potential for manipulation of multistate quantum bits in quantum information processing, for example, in designing arbitrary unitary gates.

\section{Acknowledgments}

This work has been supported by the European Union's Transfer of Knowledge project CAMEL (Grant No. MTKD-CT-2004-014427) and the Alexander von Humboldt Foundation. ESK acknowledges support from the EU Marie Curie Training Site project No. HPMTCT-2001-00294. 
[1] B.W. Shore, The Theory of Coherent Atomic Excitation (Wiley, New York, 1990).

[2] I.I. Rabi, Phys. Rev. 51, 652 (1937).

[3] L.D. Landau, Physik Z. Sowjetunion 2, 46 (1932); C. Zener, Proc. R. Soc. Lond. Ser. A 137, 696 (1932).

[4] N. Rosen and C. Zener, Phys. Rev. 40, 502 (1932).

[5] L. Allen and J. H. Eberly, Optical Resonance and TwoLevel Atoms (Dover, New York, 1987); F.T. Hioe, Phys. Rev. A 30, 2100 (1984).

[6] A. Bambini and P.R. Berman, Phys. Rev. A 23, 2496 (1981).

[7] Yu.N. Demkov and M. Kunike, Vestn. Leningr. Univ. Fiz. Khim. 16, 39 (1969); see also F.T. Hioe and C.E. Carroll, Phys. Rev. A 32, 1541 (1985); J. Zakrzewski, Phys. Rev. A 32, 3748 (1985); K.-A. Suominen and B.M. Garraway, Phys. Rev. A 45, 374 (1992).

[8] C.E. Carroll and F.T. Hioe, J. Phys. A: Math. Gen. 19, 3579 (1986).

[9] Yu.N. Demkov, Sov.Phys.-JETP 18, 138 (1964); N.V. Vitanov, J. Phys. B 26, L53 (1993), erratum ibid. 26, 2085 (1993).

[10] E.E. Nikitin, Opt. Spectrosc. 13, 431 (1962); Discuss. Faraday Soc. 33, 14 (1962); Adv. Quantum Chem. 5, 135 (1970); N.V. Vitanov, J. Phys. B 27, 1791 (1994).

[11] J.R. Morris and B.W. Shore, Phys. Rev. A 27, 906 (1983).

[12] C.P. Williams and S.H. Clearwater, Explorations in Quantum Computing, (Springer-Verlag, Berlin, 1997); A. Steane, Rep. Prog. Phys. 61, 117 (1998); M.A. Nielsen and I.L. Chuang, Quantum Computation and Quantum Information (Cambridge University Press, Cambridge, 2000).

[13] R.G. Unanyan, M. Fleischhauer, B.W. Shore, and K. Bergmann, Opt. Commun. 155, 144 (1998); H. Theuer, R.G. Unanyan, C. Habscheid, K. Klein and K. Bergmann, Optics Express 4, 77 (1999).

[14] Z. Kis and S. Stenholm, Phys. Rev. A 64, 63406 (2001).

[15] K. Bergmann, H. Theuer, and B.W. Shore, Rev. Mod. Phys. 70, 1003 (1998); N.V. Vitanov, T. Halfmann, B.W. Shore, and K. Bergmann, Ann. Rev. Phys. Chem. 52, 763 (2001); N.V. Vitanov, M. Fleischhauer, B.W. Shore and K. Bergmann, Adv. At. Mol. Opt. Phys. 46, 55 (2001).

[16] S. Stenholm, in Frontiers of Laser Spectroscopy, Les Houches Summer School Session XXVII, edited by R. Bailian, S. Haroche and S. Liberman (Amsterdam, North Holland, 1975), p. 399; R. Lefebvre and J. Savolainen, J. Chem. Phys. 60, 2509 (1974); M. Bixon and J. Jortner, J. Chem. Phys. 48, 715 (1968).

[17] N.V. Vitanov, J. Phys. B 33, 2333 (2000).

[18] M. Abramowitz and I.A. Stegun, Handbook of Mathematical Functions (Dover, New York, 1964).

[19] A.A. Rangelov, J. Piilo, and N.V. Vitanov, Phys. Rev. A 72, 053404 (2005).

[20] Y.N. Demkov and V.I. Osherov, Zh. Eksp. Teor. Fiz. 53, 1589 (1967) [Sov. Phys. JETP 26, 916 (1968)]; Y.N. Demkov and V.N. Ostrovsky, J. Phys. B 28, 403 (1995). 